\title{
Characterization of heterochromatic regions in two Triturus alpestris subspecies (Urodela: Salamandridae)
}

\author{
P. Herrero, \\ J. L. Bella $†$ and \\ B. Arano*
}

\author{
* Museo Nacional de Ciencias Naturales, \\ C/J. Gutiérrez Abascal 2. 28006 Madrid, Spain. \\ †Unidad de Genética, Departamento de Biología, \\ Facultad de Ciencias, Universidad Autónoma de \\ Madrid, 28049 Madrid, Spain.
}

The fluorescence analysis carried out in Triturus alpestris alpestris and Triturus alpestris cyreni subspecies has revealed differences related to the content and distribution of AT-rich, GC-rich and non-fluorescent heterochromatic bands. These results provide new evidence on the chromosome differentiation undergone in their phylogenetic splitting.

\section{INTRODUCTION}

Fluorescence differential staining of constitutive heterochromatic regions in the chromosomes is generally assumed to be the result of the existence of clusters of highly repetitive DNA. Biochemical analysis has demonstrated that the adeninethymine (AT) base richness induces certain fluorescent bands with some fluorochromes while the guanine-cytosine (GC) rich regions show differential fluorescence with other fluorochromes (Schmid, 1980; Schweizer, 1981). Thus these techniques are particularly useful to analyze both the DNA composition and the location of heterochromatic regions in the chromosomes.

To date ten subspecies of Triturus alpestris have been described on morphological grounds (Thorn, 1968; Dubois and Breuil, 1983). T.a. alpestris occupies almost the entire range of distribution of the species. The remaining subspecies are exclusively represented by certain isolated populations. One of these subspecies, T.a. cyreni, was first described by Wolstertorff (1932) on the basis of its restricted distribution. Its populations are only found in restricted habitats of northern Spain thus being separated from the remaining subspecies of $T$. alpestris by a large part of France.

In a previous study we reported that T.a. cyreni displays certain peculiar characteristics in the distribution of constitutive heterochromatin which are not found in T.a. alpestris (Herrero and Arano, 1986). In this paper we report the nature of these heterochromatic differences between the two sub- species of $T$. alpestris, on the basis of fluorescence banding.

\section{MATERIALS AND METHODS}

Ten adult specimens of T.a. cyreni (five $q$ and five ठ) per population were used in this study. They were collected from Lago de la Ercina, Lagos de Somiedo and Santillana del Mar (Spain). Four adult specimens of T.a. alpestris (two $q$ and two o) were collected from Bonn (FRG).

Males and females were injected with colchicine solution $(0 \cdot 1$ per cent $) 7 \mathrm{~h}$ before they were sacrificed. Pieces of intestine and testes were extracted in a hypotonic $\mathrm{KCl}(0 \cdot 1 \mathrm{M})$ solution at room temperature, they were fixed in $3: 1$ ethanol: acetic acid for $24 \mathrm{~h}$. Squash preparations were made in 45 per cent acetic acid and the coverslip was removed after freezing in liquid nitrogen. Then slides were dehydrated and subjected to the C-banding technique reported by Sumner (1972).

From each individual about 50 intestinal and testicular metaphases were studied.

The fluorescence analysis was carried out using the staining methodology developed by Schweizer (1976; 1980; and personal communication). We used chromomycin $\mathrm{A}_{3}\left(\mathrm{CMA}_{3}\right)$, Distamycin (DA) and 4'-6 diamino-2-phenylindole (DAPI) dyes supplied by SERVA. 
Fluorescence observations were carried out using a Zeiss Photo III microscope and photographs were taken with Valca 125 ASA film.

\section{RESULTS}

The two subspecies used in this study have 24 biarmed chromosomes. The C-banding pattern of both subspecies consists of centromeric bands in almost all chromosomes. Thin pericentromeric bands in variable numbers (single, double or triple) were observed in all chromosomes. Pairs 1, 3 and 8 display distal bands which affect both arms in pairs 1 and 3 and the longest arm of pair 8 . The differences between the subspecies relate to the presence in T.a. alpestris of interstitial bands in pairs 4 and 6 and a distal band in pair 12 that are absent from T.a. cyreni. On the other hand T.a. cyreni presents several bands not found in T.a. alpestris. Some are located in the centromere (pair 8 ), and others in interstitial (pairs 3 and 8) or telomeric (pairs 10 and 11) positions. Some coincident bands are larger in T.a. cyreni than in T.a. alpestris (fig. 1(a) and (1b))(Schmid et al., 1979; Herrero and Arano, 1986). We have not found inter-individual variations in banding patterns.

On the other hand, while males of T.a. alpestris posses a characteristic XY sex chromosome pair (number 4), such differentiation has not been found in T.a. cyreni (Herrero and Arano, 1986). Both males and females of T.a. cyreni present a telomeric grey C-band in the homologues of pair 4. The application of fluorochromes to both $T$. alpestris subspecies reveals new information as regards the characteristics of the C-heterochromatic regions. Three kinds of fluorescent and nonfluorescent bands have been found:

(i) AT-rich C-bands.

These regions correspond to those that are positive to DA-DAPI (Distamycin/4'-6 diamidino-2-phenylindole). Most C-bands are included in this pattern, so that the differences found between the two subspecies correspond to the absence/presence of some C-bands, particularly in the centromeric and pericentromeric regions. Good examples are the pairs 4, 6 and 8 (figs. 1 and 2).

(ii) GC-rich C-bands.

DA-CMA $\mathrm{MA}_{3}$ (Distamycin/Chromomycin $\mathrm{A}_{3}$ ) reveals very restricted areas of positive fluorescence. The positive fluorescence occurs in 5 autosomes pairs $(1,3,7,8$ and 9) in T.a. alpestris and is located in pairs 1, 3, 8,9 and 10 in T.a. cyreni. The differences between the two subspecies involve the positive $\mathrm{CMA}_{3}$ fluorescence of pairs $3,7,8$ and 10 . The differences between the 3,7 and 10 pairs are not revealed by C-banding (figs. 1 and 2).

(iii) Non fluorescent C-bands.

Such regions show a similar staining intensity to the euchromatin. This labelling has been observed in the terminal C-band of pair 12 in T.a. alpestris and in the interstitial and telomeric C-bands of pairs 3 and 11 in T.a. cyreni (figs. 1 and 2).

This analysis has not revealed more heterochromatic regions than $\mathrm{C}$-banding has.

\section{DISCUSSION}

Our results show the divergence between two isolated groups and allow us to observe the initiation of a possible phylogenetic splitting. Roger's distance reveals that these subspecies have been isolated since the Pleistocene age (Arano and Arntzen, 1987). During this time new bands in T.a. cyreni and in T.a. alpestris have emerged or have been lost (depending which state were ancestral). On the other hand, four of the heterochromatic shared bands have changed their base composition, two of them being GC-rich in T.a. alpestris and AT-rich in T.a. cyreni, and the other two being GC-rich in T.a. cyreni and AT-rich in T.a. alpestris.

Two models have been recently proposed to explain the location and growth of new heterochromatin regions. One of them (Schweizer and Loidl, 1986) predicts that the telomeres are C-band initiation sites from which there is a heterochromatin transfer to interstitial sites of other nonhomologous chromosomes during the mitotic and meiotic processes. This model predicts that a karyotype with chromosomes of similar length will tend to have telomeric C-bands rather than interstitial bands and a karyotype with chromosomes of different sizes and arm ratios will tend to possess interstitial and telomeric bands. None of these predictions are in accordance with the C-band patterns seen in the two subspecies studied as the telomeric bands are not predominant in them.

The second model (Macgregor and Sessions, 1986) proposes that C-bands would be dispersed from the heterocromatic initiation sites in the centromeres towards the pericentric region and from there to the telomeric region. Therefore, those karyotypes with more telomeric heterochromatin would have an older phylogenetic status. The slight trend observed in T.a. cyreni to have more telomeric bands than T.a. alpestris, where most 
(a)
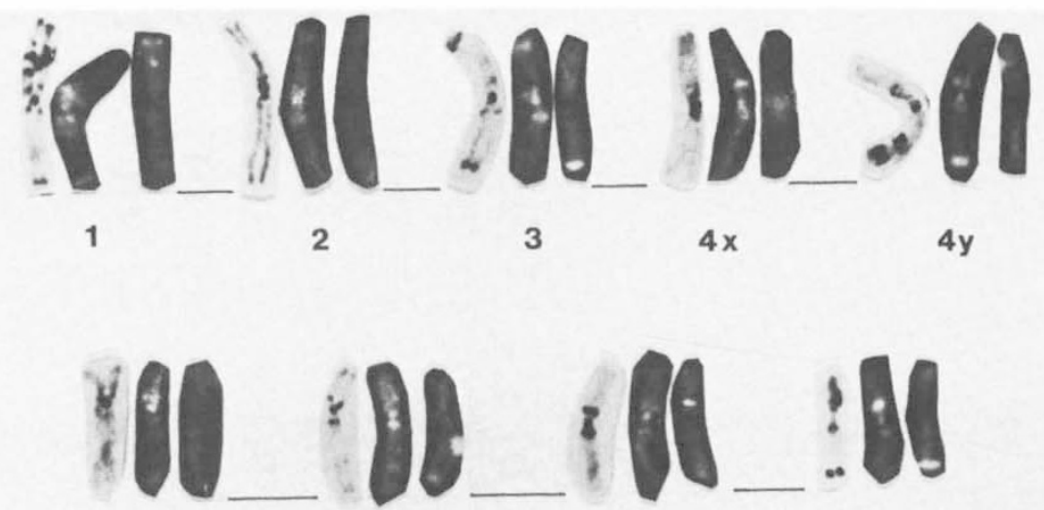

5

6

7

8
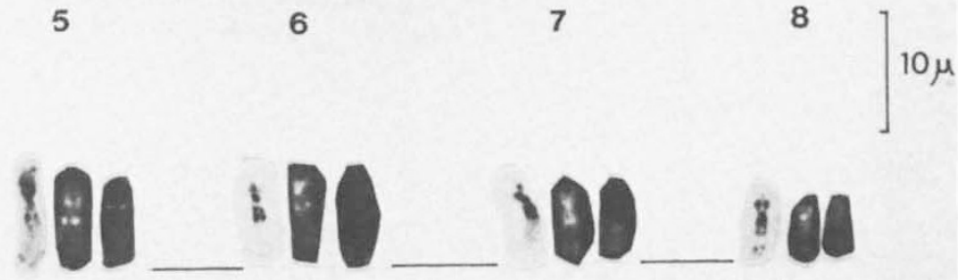

9
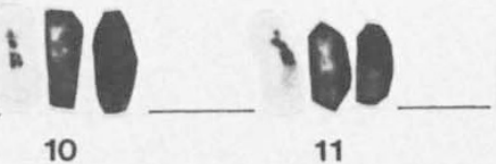

11

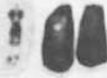

12

\section{T.a. alpestris}

(b)
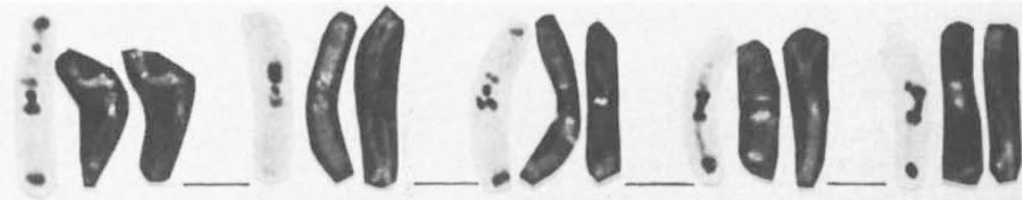

1

2

3

$4 x$

4y
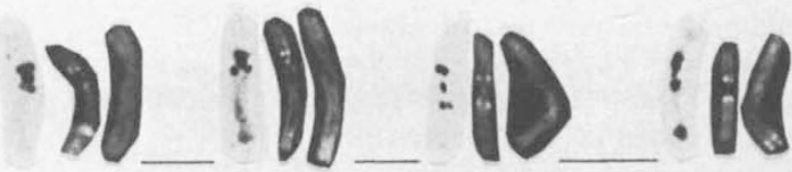

5

6

7

8
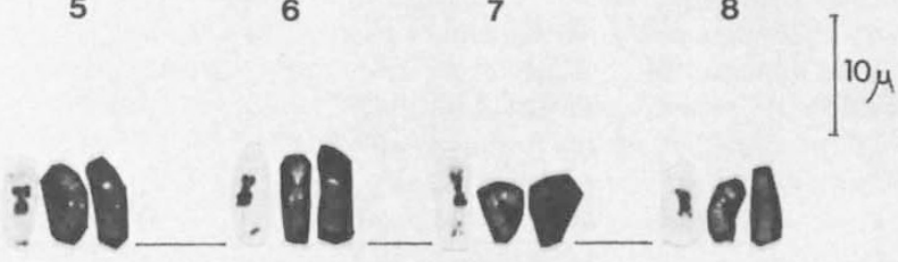

9
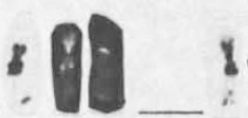

10

11

12

\section{T.a.cyreni}

Figure 1 Haploid karyotypes of: a, Triturus alpestris alpestris; b, Triturus alpestrix cyreni, after C-banding and fluorescent staining. Within each chromosomic triad, the first chromosome corresponds to the C-banding, the second one to the DA-DAPI staining and the third one to the DA-CMA 2 staining. 


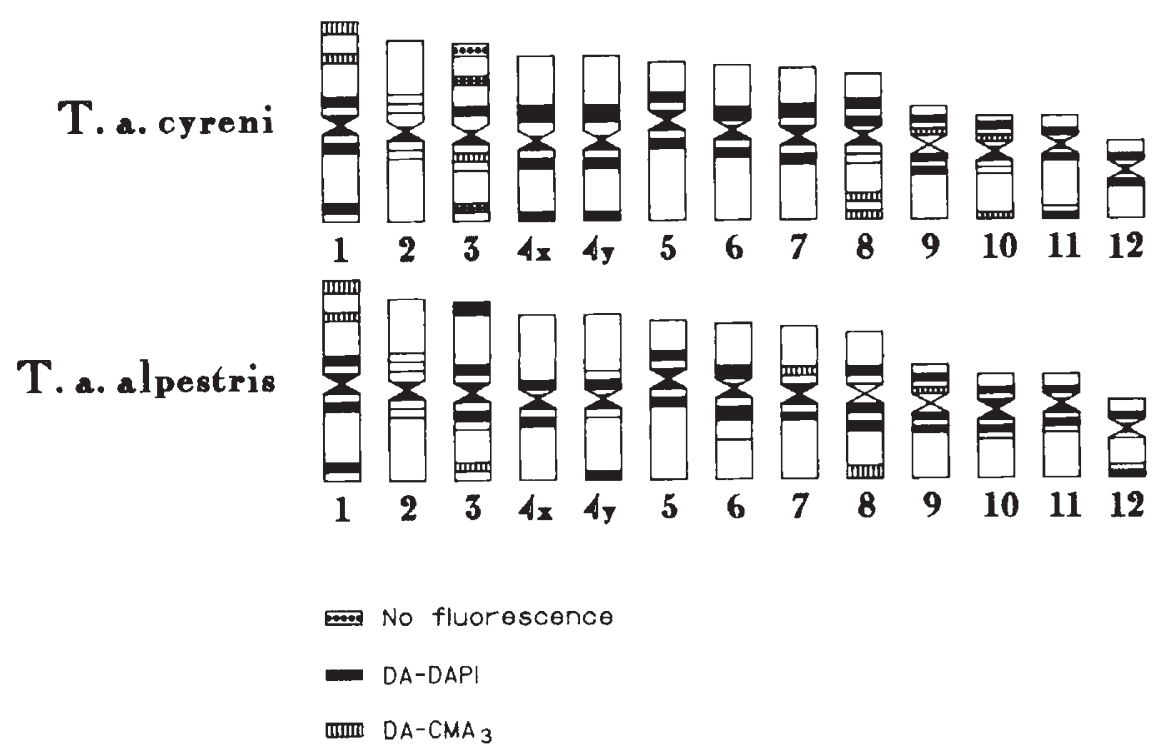

Figure 2 Idiograms of Triturus alpestris alpestris and Triturus alpestris cyreni showing the differential response to distinct fluorochromes of the C-bands contained in their chromosome complements.

bands are pericentric, would corroborate electrophoretical results which suggest that T.a. cyreni represents an older evolutionary status. However, differences in the composition of the heterochromatin of particular chromosomes might reveal a different origin for each band, which is the case for pairs 3,9 and 10 in T.a. cyreni, and pairs 3,7 and 9 in T.a. alpestris. Hence, this model would not fully explain the $T$. alpestris C-banding distribution and origin. It seems clear that chance could have played a major role in the causation of heterochromatic regions in the early stages of subspecies evolution and there is no evidence of a tendency for these regions to appear in predetermined locations in the chromosome complement.

Finally, our results from the comparative analysis of fluorescent banding show differences between the two subspecies not only in respect to the heterochromatic distribution, but also concerning the composition of several shared C-bands. Taking into account that C-banding patterns of Triturus are extremely conservative (John, 1988), the sharp differences observed between T.a. alpestris and T.a. cyreni suggest that an important divergence process may affect the $T$. alpestris complex.

Acknowledgements The authors are indebted to C. García de la Vega for his kind critical review of the original manuscript. This work has been supported in part by C.A.Y.C.I.T.-C.S.I.C (Spain) grant No. 211 and a D.A.A.D-C.S.I.C. grant.
We thank Spanish and German Nature Conservation Authorities for the authorization they provided us with to form collections.

\section{REFERENCES}

ARANO, B. AND ARNTZEN, J. W. 1987. Genetic differentiation in the alpine newt Triturus alpestris. In van Gelder, J. J., Strijbosch, H. and Bergers, P. J. M. (eds) Proc. Fourth Ord. Gen. Meet. SEH, Nijmegen 1987, Faculty of Sciences Nijmegen Press, pp. 21-24.

DUBOIS, A. AND BREUL, M. 1983. Decourverte de Triturus alpestris (Laurenti, 1768) en Calabre (Sud de l'Italie). Alytes, 2, 9-18.

HERRERO, P. AND ARANO, B. 1986. Cytogenetic and morphological studies on Triturus alpestris cyreni. In Rocek, Z. (ed.) Studies in Herpetology, Charles University Press, pp. 114-117.

JOHN, B. 1988. The biology of heterochromatin. In Verma, R. S. (ed.) Heterochromatin: Molecular and Structural Aspects, Cambridge University Press, pp. 1-147.

MACGREGOR, H. C. AND SESSIONS, S. K. 1986. The biological significance of variation in satellite DNA and heterochromatin in newts of the genus Triturus: an evolutionary perspective. Phil. Trans. R. Soc. Lond., B312, 243-259.

SCHMID, M. 1980. Chromosome banding in Amphibia. IV. Differentiation of GC and AT rich chromosome regions in Anura. Chromosoma, 77, 83-103.

SCHMID, M., OLERT, J. AND KLETT, C. 1979. Chromosome banding in Amphibia. III. Sex chromosomes in Triturus. Chromosoma, 71, 29-55.

SCHWEIZER, D. 1976. Reverse fluorescent chromosome banding with chromomycin and DAPI. Chromosoma, 58, 307324. 
SCHWEIZER, D. 1980. Simultaneous fluorescent staining of R-bands and specific heterochromatin regions DA-DAPI bands in human chromosomes. Cytogenet. Cell Genet., 27, 190-193.

SCHWEIZER, D. 1981. Counterstain enhanced chromosome banding. Hum. Genet., 57, 1-14.

SCHWEIZER, D. AND LOIDL, J. 1986. A model for heterochromatin disperson and the evolution of C-band patterns. Chromosome Today, 9, 61-74.
SUMNER, A. T. 1972. A simple technique for demonstrating centromeric heterochromatin. Exp. Cell Res., 75, 304-306. THOR N, R. 1968. Les Salamandres d'Europe, d'Asie et d'Africa du Nord. Lechevalier, Paris.

WOLTERSTORFF, w. 1932. Triturus alpestris subsp. cyreni eine neue Unterart des Bergmolches aus Nordwestspanien. Zool. Anz., Leipzig, 97, 135. 